\title{
Spontaneous emission of semiconductors in the Wigner approach
}

\author{
V S Filinov ${ }^{1}$, W Hoyer ${ }^{2}$, M Bonitz ${ }^{3}$, M Kira ${ }^{2}$, V E Fortov ${ }^{1}$ and \\ S W Koch ${ }^{2}$ \\ ${ }^{1}$ Russian Academy of Sciences, Institute for High Energy Density, Izhorskaya Street 13-19, \\ Moscow 127412, Russia \\ ${ }^{2}$ Department of Physics and Material Sciences Centre, Philipps University, Renthof 5, \\ D-35032 Marburg, Germany \\ ${ }^{3}$ Department of Physics, Rostock University, Universitätsplatz 3, D-18051 Rostock, Germany \\ E-mail: filinov@ok.ru
}

Received 3 February 2003, in final form 17 March 2003

Published 9 June 2003

Online at stacks.iop.org/JOptB/5/S299

\begin{abstract}
This paper presents a first step towards combining two well-established methods used in semiconductor physics — semiconductor Bloch equations and the Wigner approach to quantum transport. This combination provides the possibility of including spontaneous emission, i.e., the spontaneous recombination of excited electron-hole pairs in semiconductors, into the Wigner approach, which so far has been used only for systems with fixed particle number. The theory is presented and first numerical results for a three-dimensional system are shown.
\end{abstract}

Keywords: Quantum statistical computation, semiconductor, spontaneous emission, Wigner approach, Monte Carlo, quantum dynamics

\section{Introduction}

The basic elementary excitations in direct gap semiconductors such as GaAs are excited electrons in the conduction band and holes (i.e., missing electrons) in the valence band. Electronhole pairs can be optically created by the application of a short coherent laser pulse. Due to the strong Coulomb interaction and the small masses of those excited quasi-particles, a true many-body description is necessary to understand the fundamental processes. One consequence of the Coulomb interaction is the possibility that electrons and (positive) holes can form bound states, hydrogen-like bound pairs (excitons), and even larger complexes. These bound states can be coherently driven by the exciting electro-magnetic field, thus leading, e.g., to so-called excitonic resonances which are observed experimentally as macroscopic polarization. On the other hand, such polarization typically loses its phase within times of the order of a few picoseconds. That way, the polarization dephases while the electron-hole pairs remain excited. In an ideal semiconductor structure, the excited electrons and holes can only be destroyed via radiative decay, i.e., spontaneous emission from the coupling to vacuum fluctuations of the quantized light field.
After the coherences have decayed, the excited carriers form a system completely analogous to a dense (possibly partially ionized) hydrogen plasma. At sufficiently low densities and lattice temperatures, electrons and holes can form bound excitons. At higher densities or temperatures, those excitons ionize, giving rise to a correlated electronhole plasma. To treat the theoretically difficult case of strong correlations (with a Brueckner parameter $r_{s}=\bar{r} / a_{\mathrm{B}}>1$, where $\bar{r}$ is the mean interparticle distance and $a_{\mathrm{B}}$ the Bohr radius of the lowest bound exciton), in recent years quantum Monte Carlo (QMC) techniques have been developed [1-3] which allow us to obtain exact results without approximation in the many-body Coulomb interaction. However, the QMC approach is limited to thermodynamic and static properties. To get access to dynamic properties of correlated Coulomb systems an approach based on quantum dynamics in the Wigner picture has proved successful [4-9] for systems of fixed particle number near equilibrium. We know, however, that in semiconductors true thermodynamical equilibrium is typically not reached because the thermalization time after an optical excitation is comparable to the lifetimes of the excited carriers. Thus, for a realistic modelling, spontaneous emission (radiative recombination) must be included in the theory. 
During the last few years, a second class of quantum statistical and quantum kinetic methods has been developed $[10,11]$ which are very successful in the description of semiconductor heterostructures interacting with a quantized light field $[12,13]$. The main advantage of this scheme is the straightforward inclusion of any kind of interaction, as for example the coupling to a quantized light field or to a phonon reservoir. On the other hand, this scheme is limited by the necessity for an approximate treatment of the Coulomb interaction in order to decouple the infinite hierarchy of equations for the reduced density operators (BBGKY hierarchy).

The goal of this paper is to develop a theoretical and computational scheme which combines the advantages of the two approaches. The main idea is to use the experience from the quantum kinetic theory on the treatment of lightmatter interaction and radiative recombination and incorporate it into the Wigner dynamics approach. In section 2 we outline the semiconductor quantum-kinetic theory for the case of a fixed carrier number $N$ which is transformed into the Wigner representation in section 3 . Sections 4 and 5 are devoted to the extension of the theory to a variable particle number and to inclusion of the light field, respectively. Finally, section 6 shows first numerical results.

\section{Semiconductor density matrix equations}

In this section we outline the semiconductor quantum kinetic theory for the case of a fixed number $N$ of electrons and holes $[10,11]$. The Hamiltonian of the system $\mathcal{H}$ contains contributions from kinetic energy and Coulomb interaction,

$$
\begin{aligned}
\mathcal{H} & =\mathcal{H}_{0}+\sum_{\lambda=e, h} \sum_{\lambda^{\prime}=e, h} \mathcal{H}_{C}^{\lambda, \lambda^{\prime}}, \\
\mathcal{H}_{0} & =\sum_{\lambda=e, h} \int \Psi_{\lambda}^{\dagger}(x)\left(-\frac{\hbar^{2} \nabla_{x}^{2}}{2 m_{\lambda}}\right) \Psi_{\lambda}(x) \mathrm{d} x, \\
\mathcal{H}_{C}^{\lambda, \lambda^{\prime}} & =\frac{1}{2} \iint \Psi_{\lambda}^{\dagger}(x) \Psi_{\lambda^{\prime}}^{\dagger}\left(x^{\prime}\right) V\left(\left|x-x^{\prime}\right|\right) \\
& \times \Psi_{\lambda^{\prime}}\left(x^{\prime}\right) \Psi_{\lambda}(x) \mathrm{d} x \mathrm{~d} x^{\prime},
\end{aligned}
$$

where $\lambda$ and $\lambda^{\prime}$ specify electrons or holes, and $V(x)$ is the three-dimensional Coulomb matrix element. Further, $\Psi_{\lambda}^{\dagger}$ and $\Psi_{\lambda}$ are standard creation and annihilation operators in second quantization. The quantum many-body theory of this system starts from the density operator constructed from the fully antisymmetrized $N$-electron- $N$-hole wavefunctions $\left|x_{1 N}\right\rangle$,

$\hat{\rho}=\hat{\rho}_{N N}, \quad$ with

$\hat{\rho}_{N M} \equiv \frac{1}{N ! M !} \int \cdots \int \rho\left(x_{1 N} \mid y_{1 M}\right)\left|x_{1 N}\right\rangle\left\langle y_{1 M}\right| \mathrm{d} x_{1 N} \mathrm{~d} y_{1 M}$,

where we will use the short notation $x_{1 N} \equiv \begin{gathered}x_{1 N}^{e} \\ x_{1 N}^{h}\end{gathered}$, with $x_{1 N}^{e, h} \equiv x_{1}^{e, h} \cdots x_{N}^{e, h}$, for the coordinates of all electrons and holes, and the coordinate representation of $\hat{\rho}$ (the density matrix) is defined as $\left\langle x_{1 N}|\hat{\rho}| y_{1 N}\right\rangle \equiv \rho\left(x_{1 N} \mid y_{1 N}\right)=$ $\operatorname{Tr}\left[\left\{\Psi_{h}^{\dagger}\left(y_{N}^{h}\right)\right\}\left\{\Psi_{e}^{\dagger}\left(y_{N}^{e}\right)\right\}\left\{\Psi_{e}\left(x_{N}^{e}\right)\right\}\left\{\Psi_{h}\left(x_{N}^{h}\right)\right\} \hat{\rho}\right]$. Here, we introduced a short notation for complete $N$-operator products $\left\{\Psi_{e, h}\left(x_{N}\right)\right\} \equiv \Psi_{e, h}\left(x_{1}\right) \cdots \Psi_{e, h}\left(x_{N}\right)$. The equation of motion of $\hat{\rho}$ is the von Neumann (Heisenberg) equation, i $\hbar \frac{\partial}{\partial t} \hat{\rho}=$ $[\mathcal{H}, \hat{\rho}]$.
As relevant observables of the carrier system in semiconductors are usually related to single- or two-particle properties, it is convenient to introduce reduced density operators of $M$ electrons and $M^{\prime}$ holes $\left(M, M^{\prime} \leqslant N\right)$

$$
\hat{F}_{M^{\prime}}^{M}=\operatorname{Tr}_{M+1}^{N} \operatorname{Tr}_{M^{\prime}+1}^{N}[\hat{\rho}]
$$

with the matrix representation (reduced density matrix)

$$
\begin{aligned}
& F\left(\begin{array}{c|c}
x_{1 M}^{e} & y_{1 M}^{e} \\
x_{1 M^{\prime}}^{h} & y_{1 M^{\prime}}^{h}
\end{array}\right) \\
& =\operatorname{Tr}\left[\left\{\Psi_{e}^{\dagger}\left(y_{M}^{e}\right)\right\}\left\{\Psi_{h}^{\dagger}\left(y_{M^{\prime}}^{h}\right)\right\}\left\{\Psi_{h}\left(x_{M^{\prime}}^{h}\right)\right\}\left\{\Psi_{e}\left(x_{M}^{e}\right)\right\} \hat{\rho}\right],
\end{aligned}
$$

which describes correlations between $M$ electrons and $M^{\prime}$ holes, a subset of the total number of $N$ electron-hole pairs. Since the Coulomb interaction is a true many-body interaction, the equations of motion for $F_{M^{\prime}}^{M}$ are not closed, but couple to the equations involving more than $M$ electrons and $M^{\prime}$ holes, leading to the hierarchy of semiconductor density matrix equations in analogy to the familiar BBGKY hierarchy, e.g. [10],

$$
\begin{aligned}
& \left\{\mathrm{i} \hbar \frac{\partial}{\partial t}-\sum_{i=1}^{M}\left(\frac{\hbar^{2} \Delta_{y_{i}^{e}}}{2 m^{e}}-\frac{\hbar^{2} \Delta_{x_{i}^{e}}}{2 m^{e}}\right)-\sum_{j=1}^{M^{\prime}}\left(\frac{\hbar^{2} \Delta_{y_{j}^{h}}}{2 m^{h}}-\frac{\hbar^{2} \Delta_{x_{j}^{h}}}{2 m^{h}}\right)\right. \\
& -\sum_{\substack{i=1 \\
j<i}}^{M}\left[V\left(x_{i}^{e}-x_{j}^{e}\right)-V\left(y_{i}^{e}-y_{j}^{e}\right)\right] \\
& -\sum_{\substack{i=1 \\
j<i}}^{M^{\prime}}\left[V\left(x_{i}^{h}-x_{j}^{h}\right)-V\left(y_{i}^{h}-y_{j}^{h}\right)\right] \\
& \left.+\sum_{i=1}^{M} \sum_{j=1}^{M^{\prime}}\left[V\left(x_{i}^{e}-x_{j}^{h}\right)-V\left(y_{i}^{e}-y_{j}^{h}\right)\right]\right\} F\left(\begin{array}{c|c}
x_{1 M}^{e} & y_{1 M}^{e} \\
x_{1 M^{\prime}}^{h} & y_{1 M^{\prime}}^{h}
\end{array}\right) \\
& =\int\left(\sum_{i=1}^{M}\left[V\left(x_{i}^{e}-x\right)-V\left(y_{i}^{e}-x\right)\right]\right. \\
& \left.-\sum_{j=1}^{M^{\prime}}\left[V\left(x_{j}^{h}-x\right)-V\left(y_{j}^{h}-x\right)\right]\right) \\
& \times\left[F\left(\begin{array}{c|c}
x_{1 M}^{e}, x & y_{1 M}^{e}, x \\
x_{1 M^{\prime}}^{h} & y_{1 M^{\prime}}^{h}
\end{array}\right)-F\left(\begin{array}{c|c}
x_{1 M}^{e} & y_{1 M}^{e} \\
x_{1 M^{\prime}}^{h}, x & y_{1 M^{\prime}}^{h}, x
\end{array}\right)\right] \mathrm{d} x .
\end{aligned}
$$

This set of equations provides the dynamics for all reduced density matrices of a system with two types of carrier experiencing Coulomb interaction. These equations are similar to the semiconductor Bloch equations [11]. Note that mean-field (Hartree-Fock) contributions are contained in the terms on the right-hand side (rhs). This hierarchy naturally terminates with the coupling to the full $N$-pair density matrix, the equation of which (the von Neumann equation), obviously, has a zero rhs.

Quantum kinetic theories in general, and approaches based on the semiconductor Bloch equations in particular, introduce truncations of the hierarchy to make the problem computationally feasible. Of course, this always leads to the neglect of certain Coulomb correlation effects. In the next section we develop an alternative approach which avoids any truncation. 


\section{N-body quantum dynamics in the Wigner representation}

For classical systems, an exact treatment of many-body correlations is possible and successfully realized by molecular dynamics (MD) where the only approximation is the mapping of the $N$-body system onto a similar one with a tractable smaller number $N_{\text {sim }}$, for a discussion see e.g. [10]. Such a classical approach is, however, not adequate for the intrinsically quantum dynamics of semiconductors. We, therefore, develop in the following a quantum MD scheme which overcomes these shortcomings and, at the same time, is capable of treating many-body correlations exactly. The basis is the Wigner representation of the von Neumann equation, the Wigner-Liouville equation (WLE).

To derive the WLE for the full density matrix $\rho\left(x_{1 N} \mid y_{1 N}\right)$ we introduce centre of mass and relative coordinates in the standard manner, $\boldsymbol{R} \equiv \boldsymbol{R}_{1 N} \equiv\left(\boldsymbol{x}_{1 N}+\boldsymbol{y}_{1 N}\right) / 2$ and $\boldsymbol{r} \equiv \boldsymbol{r}_{1 N} \equiv$ $\boldsymbol{x}_{1 N}-\boldsymbol{y}_{1 N}$. The Wigner distribution function (WF) is defined by [14]

$$
f(\boldsymbol{p}, \boldsymbol{R}, t)=\frac{1}{(2 \pi \hbar)^{6 N}} \int \rho\left(\boldsymbol{R}-\frac{\boldsymbol{r}}{2}, \boldsymbol{R}+\frac{\boldsymbol{r}}{2}\right) \mathrm{e}^{\mathrm{i} p \boldsymbol{r} / \hbar} \mathrm{d} \boldsymbol{r} .
$$

Using this definition it is straightforward to obtain the Wigner transform of equation (5) for the full density matrix $[10,14]$ $\frac{\partial f}{\partial t}+\frac{\boldsymbol{p}}{m} \frac{\partial f}{\partial \boldsymbol{r}}-\frac{\partial V(r)}{\partial \boldsymbol{r}} \frac{\partial f}{\partial \boldsymbol{p}}=\int_{-\infty}^{\infty} \mathrm{d} s f(\boldsymbol{p}-\boldsymbol{s}, \boldsymbol{r}, t) \omega(\boldsymbol{s}, \boldsymbol{r})$,

where

$$
\begin{array}{r}
\omega(s, \boldsymbol{q})=\boldsymbol{F}(\boldsymbol{q}) \frac{\mathrm{d} \delta(s)}{\mathrm{d} s}+\frac{4}{\hbar(2 \pi \hbar)^{6 N}} \\
\times \int \mathrm{d} \overline{\boldsymbol{q}} V(q-\bar{q}) \sin \left(\frac{2 s \overline{\boldsymbol{q}}}{\hbar}\right),
\end{array}
$$

and $\boldsymbol{F}(\boldsymbol{q})=-\partial V(q) / \partial \boldsymbol{q}$ is the classical force. Obviously, the force term in $\omega$ exactly cancels the last term on the lefthand side (lhs) of equation (7). Retaining these terms allows us to write the WLE as the classical Liouville equation (lhs of equation (7)) plus a quantum correction (all terms on the rhs of equation (7)) which vanish for $\hbar \rightarrow 0$. This form allows us to identically transform equation (7) into an integral equation [4],

$$
\begin{aligned}
& f(\boldsymbol{p}, \boldsymbol{q}, t)=f_{0}\left(\boldsymbol{p}_{\mathbf{o}}, \boldsymbol{q}_{\mathbf{o}}\right) \\
& \quad+\int_{0}^{t} \mathrm{~d} \tau \int_{-\infty}^{\infty} \mathrm{d} s f\left(\boldsymbol{p}_{\tau}-\boldsymbol{s}, \boldsymbol{p}_{\tau}, \tau\right) \omega\left(\boldsymbol{s}, \boldsymbol{q}_{\tau}\right)
\end{aligned}
$$

where $\left\{\boldsymbol{q}_{\tau}(\tau ; \boldsymbol{p}, \boldsymbol{q}, t), \boldsymbol{p}_{\tau}(\tau ; \boldsymbol{p}, \boldsymbol{q}, t)\right\}$ is the classical dynamic $\boldsymbol{p} \boldsymbol{q}$-trajectory with initial conditions at $\tau=t$ in points $\boldsymbol{p}, \boldsymbol{q}$ and evolving backwards in time up to time $\tau$ (solutions of the Hamilton equations associated to the WLE)

$$
\begin{aligned}
\mathrm{d} \boldsymbol{p}_{\tau} / \mathrm{d} \tau=\boldsymbol{F}\left(\boldsymbol{q}_{\tau}\right) ; & \boldsymbol{q}_{t}(t ; \boldsymbol{p}, \boldsymbol{q}, t)=\boldsymbol{q} ; \\
\mathrm{d} \boldsymbol{q}_{\tau} / \mathrm{d} \tau=\boldsymbol{p}_{\tau} / 2 m ; & \boldsymbol{p}_{t}(t ; \boldsymbol{p}, \boldsymbol{q}, t)=\boldsymbol{p} .
\end{aligned}
$$

The first term of equation (9) is the coherent sum of positive and negative valued contributions of these trajectories related to the initial WF $f_{0}$. This WF should be taken at arguments $p_{0}$ and $q_{0}$, i.e. the trajectories at time $\tau=0$, and contains all powers of Plank's constant. Notice that even the first term may describe the evolution of a quantum many-body state if the initial WF $f_{0}(p, q)$ is chosen appropriately. The integral term in equation (9) describes the perturbation of the classical trajectories due to quantum effects, for details we refer to [4].

The structure of equation (9) suggests constructing its solution iteratively, starting with $f_{0}$. Let us, therefore, rewrite equation (9) in the following compact form, $f^{t}=f_{0}^{t}+K_{\tau}^{t} f^{\tau}$, where the superscript on the WF denotes the time argument and $K_{\tau_{1}}^{\tau_{2}}$ denotes the time integral in equation (9) with $\tau_{2}$ and $\tau_{1}$ indicating the upper and lower integration limits. Then, the iteration series has the form [4-9]

$$
f^{t}=f_{0}^{t}+K_{\tau_{1}}^{t} f_{0}^{\tau_{1}}+K_{\tau_{2}}^{t} K_{\tau_{1}}^{\tau_{2}} f_{0}^{\tau_{1}}+K_{\tau_{3}}^{t} K_{\tau_{2}}^{\tau_{3}} K_{\tau_{1}}^{\tau_{2}} f_{0}^{\tau_{1}}+\cdots,
$$

where the first term, as we mentioned before, describes the classical evolution of an initial (classical or quantum) WF $f_{0}$ (it may contain any order of Planck's constant). The remaining terms systematically account for all dynamic quantum corrections (trajectories with momentum jumps, $s$, arising from the shifted momentum arguments in the WF under the integral in equation (7)) including, e.g., tunnelling effects and correctly accounting for the Heisenberg uncertainty principle. Thus, the solution of equation (11) can be understood as a properly weighted sum of classical and quantum phase space trajectories [4-9]

Using the solution $f^{t}$ we can compute averages of arbitrary operators in the standard way and obtain any dynamic macroscopic property of the correlated electron-hole system without approximations on the Coulomb interaction. Naturally, the true particle number $N$ is replaced by a greatly reduced number $N_{\text {sim }}$ which is of the order 50-100. The solution scheme is a combination of QMC and classical MD methods: QMC is used to generate the correlated initial state, MD generates the classical $p-q$ trajectories and Monte Carlo (MC) methods are applied to perform an important sampling of the dominant terms of the iteration series [15], see also section 6

\section{Variable number of particles. Recombination of electron hole pairs}

In order to include recombination effects as discussed in the introduction, the above Wigner dynamics scheme has to be generalized to the case of variable electron and hole numbers. The corresponding generalization of the density operator (3) is

$$
\begin{gathered}
\hat{\rho}=\oplus_{M, L} \hat{\rho}_{L M}, \\
\operatorname{Tr} \hat{\rho} \equiv \sum_{M}\left[\frac{1}{M !} \int \cdots \int \rho\left(x_{1 M} \mid x_{1 M}\right) \mathrm{d} x_{1 M}\right]=1
\end{gathered}
$$

where $\oplus$ denotes the direct sum over Hilbert spaces with different numbers of particles.

The case of a variable particle number makes the definition of reduced density matrices slightly more cumbersome, since now few-particle expectation values contain contributions from all higher-order statistical operators

$$
\begin{aligned}
& F\left(x_{1 L} \mid y_{1 M}\right) \equiv \operatorname{Tr}\left[\left\{\Psi^{\dagger}\left(y_{M}\right)\right\}\left\{\Psi\left(x_{L}\right)\right\} \hat{\rho}\right] \\
& =\sum_{s=0}^{N-L} \frac{1}{s !} \int \cdots \int \rho\left(x_{1 L}, z_{1 s} \mid y_{1 M}, z_{1 s}\right) \mathrm{d} z_{1 s}
\end{aligned}
$$


Here $N$ is again the total (maximum available) number of particles, so, obviously, $L+s \leqslant N$ and $M+s \leqslant N$ and, without loss of generality, we assume $M \leqslant L$. Only in the trivial case that $L=M=N$ (only the term with $s=0$ contributes and no integrations are performed) is $F\left(x_{1 L} \mid y_{1 M}\right)=\rho\left(x_{1 L} \mid y_{1 M}\right)$. In all other cases $F\left(x_{1 L} \mid y_{1 M}\right)$ contains contributions from additional particle complexes larger than $M$.

To use these results within the Wigner dynamics approach, we also need the inverse relation which, after some algebra, is found to be

$$
\begin{aligned}
& \rho\left(x_{1 L} \mid y_{1 M}\right) \\
& =\sum_{s=0}^{N-L}(-1)^{s} \frac{1}{s !} \int \cdots \int F\left(x_{1 L}, z_{1 s} \mid y_{1 M}, z_{1 s}\right) \mathrm{d} z_{1 s} .
\end{aligned}
$$

The generalization to the case of two types of particle is straightforward.

\section{Coupling of carriers to the light field}

In this section, we present first results of how to include the coupling to a quantized light field in a Wigner dynamics formalism. This method provides a microscopic description of the effect of spontaneous emission where, for sake of clarity, we include Coulomb effects on the spontaneous emission at the simplest possible level. As a result, the system Hamiltonian (2) acquires two additional contributions, $\mathcal{H}_{D}$ and $\mathcal{H}_{\mathrm{em}}$. The lightmatter interaction is described by

$$
\mathcal{H}_{D}=-\frac{1}{\varepsilon_{0}} \int \Psi_{h}(x) \Psi_{e}(x) \boldsymbol{D}(x) \cdot \boldsymbol{d}_{\mathrm{cv}}^{*} \mathrm{~d} x+\text { h.c. },
$$

with the mode expansion of the quantized electric field [12] $\frac{1}{\varepsilon_{0}} \boldsymbol{D}(x)=\sum_{q} \hat{e}_{q}\left[\mathcal{F}_{q}(x) B_{q}+\right.$ h.c. $]$, where we denote $\mathcal{F}_{q}(x)=$ $\mathrm{i} \mathcal{E}_{q} \mathrm{e}^{\mathrm{i} q \cdot x}, \boldsymbol{d}_{\mathrm{cv}}$ is the interband dipole matrix element, $\hat{e}_{q}$ is the polarization vector of the field mode and $\mathcal{E}_{q}$ the vacuum field amplitude [16]. The dynamics of the free electro-magnetic field is given by the Hamiltonian $\mathcal{H}_{\mathrm{em}}=\sum_{q} \hbar \omega_{q}\left(B_{q}^{\dagger} B_{q}+\frac{1}{2}\right)$.

The light-matter interaction contribution to the equation of motion of any reduced density operator $\hat{F}_{M^{\prime}}^{M}$, equation (4), is given by the commutator $\left[\hat{F}_{M^{\prime}}^{M}, \mathcal{H}_{D}\right]$ which transforms into two additional terms on the rhs of the semiconductor density matrix equations (5) of the form $A(x \mid y)-[A(y \mid x)]^{*} \equiv A_{D}$. The result for the matrix $A$ is

$$
\begin{aligned}
A\left(\begin{array}{c|c}
x_{1 M}^{e} & y_{1 M}^{e} \\
x_{1 M^{\prime}}^{h} & y_{1 M^{\prime}}^{h}
\end{array}\right)=-\frac{\boldsymbol{d}_{\mathrm{cv}}^{*}}{\varepsilon_{0}} \sum_{i=1}^{M}(-1)^{i} \sum_{j=1}^{M^{\prime}}(-1)^{M^{\prime}-j} \delta\left(y_{i}^{e}-y_{j}^{h}\right) \\
\quad \times\left\langle\left\{\Psi_{e}^{\dagger}\left(y_{M}^{e}\right)\right\}_{i}^{\prime}\left\{\Psi_{h}^{\dagger}\left(y_{M^{\prime}}^{h}\right)\right\}_{j}^{\prime}\left\{\Psi_{h}\left(x_{M^{\prime}}^{h}\right)\right\}\left\{\Psi_{e}\left(x_{M}^{e}\right)\right\} \boldsymbol{D}\left(y_{i}^{e}\right)\right\rangle \\
\quad-\frac{\boldsymbol{d}_{\mathrm{cv}}^{*}}{\varepsilon_{0}} \sum_{i=1}^{M}(-1)^{i}\left\langle\left\{\Psi_{e}^{\dagger}\left(y_{M}^{e}\right)\right\}_{i}^{\prime}\left\{\Psi_{h}^{\dagger}\left(y_{M^{\prime}}^{h}\right)\right\}\left\{\Psi_{h}\left(x_{M^{\prime}}^{h}\right)\right\}\right. \\
\left.\quad \times \Psi_{h}\left(y_{i}^{e}\right)\left\{\Psi_{e}\left(x_{M}^{e}\right)\right\} \boldsymbol{D}\left(y_{i}^{e}\right)\right\rangle-\frac{\boldsymbol{d}_{\mathrm{cv}}^{*}}{\varepsilon_{0}} \sum_{j=1}^{M^{\prime}}(-1)^{M^{\prime}+M-j+1} \\
\quad \times\left\langle\left\{\Psi_{e}^{\dagger}\left(y_{M}^{e}\right)\right\}\left\{\Psi_{h}^{\dagger}\left(y_{M^{\prime}}^{h}\right)\right\}_{j}^{\prime}\left\{\Psi_{h}\left(x_{M^{\prime}}^{h}\right)\right\}\left\{\Psi_{e}\left(x_{M}^{e}\right)\right\}\right. \\
\left.\quad \times \Psi_{e}\left(y_{j}^{h}\right) \boldsymbol{D}\left(y_{j}^{h}\right)\right\rangle,
\end{aligned}
$$

where we introduce the short notation $\left\{\Psi\left(y_{M}\right)\right\}_{i}^{\prime}$ for a product of operators in its original order with the $i$ th operator missing, and $\langle\cdots\rangle$ from now on denotes averaging with the full density operator $\hat{\rho}$.

Obviously, the light-matter interaction couples expectation values over pure carrier operator products to mixed products between carrier and photon operators. In order to eliminate those photon assisted terms, we have to establish the equations of motion for them, solve them adiabatically and eliminate the photon degrees of freedom. Therefore we have to set up the equation of motion for a general operator of the form

$$
\left\langle B_{q}^{\dagger}\left\{\Psi_{e}^{\dagger}\left(y_{M}^{e}\right)\right\}\left\{\Psi_{h}^{\dagger}\left(y_{M^{\prime}}^{h}\right)\right\}\left\{\Psi_{h}\left(x_{M^{\prime}+1}^{h}\right)\right\}\left\{\Psi_{h}\left(x_{M+1}^{e}\right)\right\}\right\rangle,
$$

i.e., for terms where one pair of electron and hole creation operators is replaced by a photon operator. Here various approximations are possible. In the general solution,

$$
\begin{aligned}
\mathrm{i} \hbar & \frac{\partial}{\partial t}\left\langle B_{q}^{\dagger}\left\{\Psi_{e}^{\dagger}\left(y_{M}^{e}\right)\right\}\left\{\Psi_{h}^{\dagger}\left(y_{M^{\prime}}^{h}\right)\right\}\left\{\Psi_{h}\left(x_{M^{\prime}+1}^{h}\right)\right\}\left\{\Psi_{h}\left(x_{M+1}^{e}\right)\right\}\right\rangle \\
= & \left(\sum_{i=1}^{M+1} \frac{-\hbar^{2} \Delta_{x_{i}^{e}}}{2 m^{e}}-\sum_{i=1}^{M} \frac{-\hbar^{2} \Delta_{y_{i}^{e}}}{2 m^{e}}\right. \\
& \left.+\sum_{j=1}^{M^{\prime}+1} \frac{-\hbar^{2} \Delta_{x_{j}^{h}}}{2 m^{h}}-\sum_{j=1}^{M^{\prime}} \frac{-\hbar^{2} \Delta_{x_{j}^{h}}}{2 m^{h}}-\hbar \omega_{q}\right) \\
& \times\left\langle B_{q}^{\dagger}\left\{\Psi_{e}^{\dagger}\left(y_{M}^{e}\right)\right\}\left\{\Psi_{h}^{\dagger}\left(y_{M^{\prime}}^{h}\right)\right\}\left\{\Psi_{h}\left(x_{M^{\prime}+1}^{h}\right)\right\}\left\{\Psi_{h}\left(x_{M+1}^{e}\right)\right\}\right\rangle \\
& +\int \mathrm{d} z \mathcal{F}_{q}(z) d_{\mathrm{cv}}\left\langle\Psi_{e}^{\dagger}(z) \Psi_{h}^{\dagger}(z)\left\{\Psi_{e}^{\dagger}\left(y_{M}^{e}\right)\right\}\left\{\Psi_{h}^{\dagger}\left(y_{M^{\prime}}^{h}\right)\right\}\right. \\
& \left.\times\left\{\Psi_{h}\left(x_{M^{\prime}+1}^{h}\right)\right\}\left\{\Psi_{h}\left(x_{M+1}^{e}\right)\right\}\right\rangle+\Omega_{\mathrm{Stim}}+\Omega_{\mathrm{Coul}},
\end{aligned}
$$

the stimulated contribution $\Omega_{\text {Stim }}$ contains terms proportional to the photon number $\left\langle B_{q}^{\dagger} B_{q}\right\rangle$ and is negligible for the case of weak recombination. The Coulomb contribution $\Omega_{\text {Coul }}$ introduces the Coulombic resonance into the equation of the photon-assisted terms. Both contributions are neglected in the present approach.

After some lengthy transformations in momentum space we obtain for the photon-assisted terms,

$$
\begin{aligned}
& -\frac{\boldsymbol{d}_{\mathrm{cv}}}{\varepsilon_{0}}\left\langle\boldsymbol{D}(z)\left\{\Psi_{e}^{\dagger}\left(y_{M}^{e}\right)\right\}\left\{\Psi_{h}^{\dagger}\left(y_{M^{\prime}}^{h}\right)\right\}\left\{\Psi_{h}\left(x_{M^{\prime}+1}^{h}\right)\right\}\left\{\Psi_{h}\left(x_{M+1}^{e}\right)\right\rangle\right. \\
& \approx \\
& \quad \frac{1}{\mathcal{V}} \pi \mathrm{i} \sum_{q, k}\left|\mathcal{E}_{q}\right|^{2}\left|d_{\mathrm{cv}}\right|^{2} \delta\left(\frac{\hbar^{2} q^{2}}{2 M}+\frac{\hbar^{2} k^{2}}{2 \mu}+E_{G}-\hbar \omega_{q}\right) \\
& \quad \times \iint\left\langle\Psi_{e}^{\dagger}\left(z_{h}\right) \Psi_{h}^{\dagger}\left(z_{e}\right)\left\{\Psi_{e}^{\dagger}\left(y_{M}^{e}\right)\right\}\left\{\Psi_{h}^{\dagger}\left(y_{M^{\prime}}^{h}\right)\right\}\right. \\
& \quad \times\left\{\Psi_{h}\left(x_{M^{\prime}+1}^{h}\right)\right\}\left\{\Psi_{h}\left(x_{M+1}^{e}\right)\right\rangle \mathrm{e}^{-\mathrm{i} k r} \mathrm{e}^{-\mathrm{i} q R} \mathrm{~d} R \mathrm{~d} r,
\end{aligned}
$$

where the total mass $M=m_{e}+m_{h}$ and the reduced mass $\frac{1}{\mu}=\frac{1}{m_{e}}+\frac{1}{m_{h}}$ have been introduced. Furthermore, the integration variables have been chosen as shifted centre of mass $R=\frac{m_{e} y^{e}+m_{h} y^{h}}{M}-z$ and relative coordinate $r=y^{h}-y^{e}$. The arguments $z_{e} \equiv z-R+r^{e}$ and $z_{h} \equiv z-R-r^{h}$ are defined with $r^{e(h)}=\frac{m_{e(h)}}{M}$. This equation is valid as long as recombination out of the continuum is dominant, otherwise Coulomb interaction in the photon-assisted terms has to be taken into account. Equation (19) reflects this fact via the energy $\delta$-function, where only centre of mass and relative momenta of free electron-hole pairs enter.

In order to obtain a simpler equation, we first note that, due to the small photon momentum, the $\delta$-function contributes most for very small centre of mass momenta. Therefore, we 
may drop the exponential factor $\mathrm{e}^{-\mathrm{i} q R}$ and approximate the original $k$-dependent dephasing by a constant,

$$
\begin{aligned}
\gamma_{k} & =\pi \sum_{q}\left|\mathcal{E}_{q}\right|^{2}\left|d_{\mathrm{cv}}\right|^{2} \delta\left(\frac{\hbar^{2} q^{2}}{2 M}+\frac{\hbar^{2} k^{2}}{2 \mu}+E_{G}-\hbar \omega_{q}\right) \\
& \approx \pi \sum_{q}\left|\mathcal{E}_{q}\right|^{2}\left|d_{\mathrm{cv}}\right|^{2} \delta\left(\hbar \omega_{q}-E_{G}\right) \\
& =\frac{1}{4 \pi}\left(\frac{E_{G}}{\hbar c}\right)^{3} \frac{\left|d_{\mathrm{cv}}\right|^{2}}{\epsilon_{0}} \equiv \gamma_{\mathrm{rad}} \approx 3.5 \times 10^{-5} E_{\mathrm{B}},
\end{aligned}
$$

where $E_{\mathrm{B}}=4.2 \mathrm{meV}$ is the $3 \mathrm{D}$ exciton binding energy. We thus obtain

$$
\begin{aligned}
& -\frac{\boldsymbol{d}_{\mathrm{cv}}}{\varepsilon_{0}}\left\langle\boldsymbol{D}(z)\left\{\Psi_{e}^{\dagger}\left(y_{M}^{e}\right)\right\}\left\{\Psi_{h}^{\dagger}\left(y_{M^{\prime}}^{h}\right)\right\}\left\{\Psi_{h}\left(x_{M^{\prime}+1}^{h}\right)\right\}\left\{\Psi_{h}\left(x_{M+1}^{e}\right)\right\rangle\right. \\
& \approx \mathrm{i} \gamma_{\mathrm{rad}} \int\left\langle\Psi_{e}^{\dagger}(R) \Psi_{h}^{\dagger}(R) \cdots\right\rangle \mathrm{d} R .
\end{aligned}
$$

Approximation (20) corresponds to the requirement that electron and hole must be at the same position when recombining. Setting $q$ equal to zero has its counterpart in the non-locality of the recombination, i.e., the centre-of-mass position $R$ of the electron-hole pair does not have to coincide with the photon coordinate $z$. From equation (20), we can calculate the corresponding decay constant: $\tau_{\text {rad }}=\hbar / \gamma_{\text {rad }} \approx$ $4.5 \mathrm{~ns}$.

We insert the result of equation (21) into equation (16) to eliminate the photon field and to get an effective equation for the carrier dynamics alone which takes into account the microscopic nature of the spontaneous emission. Thus we obtain for the full recombination contribution to the semiconductor density matrix equations

$$
\begin{aligned}
& A_{D}\left(\begin{array}{c|c}
x_{1 M}^{e} & y_{1 M}^{e} \\
x_{1 M^{\prime}}^{h} & y_{1 M^{\prime}}^{h}
\end{array}\right)=-\mathrm{i} \gamma_{\mathrm{rad}} \sum_{i=1}^{M} \sum_{j=1}^{M^{\prime}} \delta\left(y_{i}^{e}-y_{j}^{h}\right) \\
& \times \int \mathrm{d} z F\left(\begin{array}{c|c}
x_{1 M}^{e} & y_{1 M}^{e}, y_{i}^{e} \rightarrow z \\
x_{1 M^{\prime}}^{h} & y_{1 M^{\prime}}^{h}, y_{j}^{h} \rightarrow z
\end{array}\right) \\
& -\mathrm{i} \gamma_{\mathrm{rad}} \sum_{i=1}^{M} \int \mathrm{d} z F\left(\begin{array}{c|c}
x_{1 M}^{e} & y_{1 M}^{e}, y_{i}^{e} \rightarrow z \\
x_{1 M^{\prime}}^{h}, y_{i}^{e} & y_{1 M^{\prime}}^{h}, z
\end{array}\right) \\
& -\mathrm{i} \gamma_{\text {rad }} \sum_{j=1}^{M^{\prime}} \int \mathrm{d} z F\left(\begin{array}{c|c}
x_{1 M}^{e}, y_{j}^{h} & y_{1 M}^{e}, z \\
x_{1 M^{\prime}}^{h} & y_{1 M^{\prime}}^{h}, y_{j}^{h} \rightarrow z
\end{array}\right) \\
& -\mathrm{i} \gamma_{\mathrm{rad}} \sum_{i=1}^{M} \sum_{j=1}^{M^{\prime}} \delta\left(x_{i}^{e}-x_{j}^{h}\right) \\
& \times \int \mathrm{d} z F\left(\begin{array}{c|c}
x_{1 M}^{e}, x_{i}^{e} \rightarrow z & y_{1 M}^{e} \\
x_{1 M^{\prime}}^{h}, x_{j}^{h} \rightarrow z & y_{1 M^{\prime}}^{h}
\end{array}\right) \\
& -\mathrm{i} \gamma_{\operatorname{rad}} \sum_{i=1}^{M} \int \mathrm{d} z F\left(\begin{array}{c|c}
x_{1 M}^{e}, x_{i}^{e} \rightarrow z & y_{1 M}^{e} \\
x_{1 M^{\prime}}^{h}, z & y_{1 M^{\prime}}^{h}, x_{i}^{e}
\end{array}\right) \\
& -\mathrm{i} \gamma_{\text {rad }} \sum_{j=1}^{M^{\prime}} \int \mathrm{d} z F\left(\begin{array}{c|c}
x_{1 M}^{e}, z & y_{1 M}^{e}, x_{j}^{h} \\
x_{1 M^{\prime}}^{h}, x_{j}^{h} \rightarrow z & y_{1 M^{\prime}}^{h}
\end{array}\right),
\end{aligned}
$$

where the short notation should be self-explanatory. Arrows indicate that the given coordinate is replaced by the integration variable $z$. While the first and fourth term on the rhs invoke only density matrices with the same number of carriers as the one on the left, the remaining four terms reveal in which way light-matter interaction couples density matrices with different numbers of carriers. The first and fourth term are, therefore, the only terms which are present in the equation of motion of the full density matrix. Using these results, we may now return to the dynamic equation for the Wigner function of $M$ electrons and $M^{\prime}$ holes $f_{M^{\prime}}^{M}$. This involves use of relation (14) and incorporation of the recombination contributions given by equation (22) yielding

$$
\begin{aligned}
f_{M^{\prime}}^{M} & (p, q, t)=f_{M^{\prime} 0}^{M}\left(p_{0}, q_{0}\right) \\
& +\int_{0}^{t} \mathrm{~d} \tau \int_{-\infty}^{\infty} \mathrm{d} s f_{M^{\prime}}^{M}\left(p_{\tau}-s, q_{\tau}, \tau\right) \omega\left(s, q_{\tau}\right) \\
& -\frac{\mathrm{i} 2 \gamma_{\mathrm{rad}}}{\hbar(2 \pi \hbar)^{3}} \sum_{j=1}^{M} \sum_{k=1}^{M^{\prime}} \int_{0}^{t} \mathrm{~d} \tau \int_{-\infty}^{\infty} \mathrm{d} X \mathrm{~d} s \\
& \times \cos \left(\frac{s\left(q_{e \tau}^{j}-q_{h \tau}^{k}\right)}{\hbar}-\frac{X\left(p_{e \tau}^{j}+p_{h \tau}^{k}\right)}{\hbar}\right) \\
& \times f_{M^{\prime}}^{M}\left(\bar{p}_{e \tau}, p_{e \tau}^{j}+\frac{s}{2} ; \bar{p}_{h \tau}, p_{h \tau}^{k}-\frac{s}{2} ;\right. \\
& \left.\bar{q}_{e \tau}, q_{e \tau}^{j}+\frac{X}{2} ; \bar{q}_{h \tau}, q_{h \tau}^{k}+\frac{X}{2} ; \tau\right) \\
& -\frac{\mathrm{i} 2 \gamma_{\mathrm{rad}}}{\hbar(2 \pi \hbar)^{6}} \sum_{j=1}^{M} \int_{0}^{t} \mathrm{~d} \tau \int_{-\infty}^{\infty} \mathrm{d} z \mathrm{~d} \tilde{z} \mathrm{~d} s_{e}^{j} \mathrm{~d} s_{h} \\
& \times \cos \left(\frac{\left(\tilde{p}_{h \tau}+p_{e \tau}^{j}\right) \tilde{z}}{\hbar}-\frac{\left(z s_{e}^{j}+\tilde{z} s_{h}\right)}{\hbar}\right) \\
& \times f_{M^{\prime}}^{M+1}\left(p_{e \tau}, \tilde{p}_{e \tau}-\frac{s_{e}}{2} ; \bar{p}_{h \tau}, p_{h \tau}^{k}-\frac{s_{h}^{k}}{2} ; \bar{q}_{h \tau}, q_{h \tau}^{k}-\frac{\tilde{z}}{2} ; \tau\right) \\
& \times f_{M^{\prime}+1}^{M}\left(\bar{p}_{e \tau}, p_{e \tau}^{j}-\frac{s_{e}^{j}}{2} ; \bar{p}_{h \tau}, \tilde{p}_{h \tau}-\frac{s_{h}}{2}\right. \\
& \left.\bar{q}_{e \tau}, q_{e \tau}^{j}-\frac{\tilde{z}}{2} ; \bar{q}_{h \tau}, q_{e \tau}^{j}+\frac{z}{2} ; \tau\right) \\
& -\frac{\mathrm{i} 2 \gamma_{\mathrm{rad}}}{\hbar(2 \pi \hbar)^{6}} \sum_{k=1}^{M^{\prime}} \int_{0}^{t} \mathrm{~d} \tau \int_{-\infty}^{\infty} \mathrm{d} z \mathrm{~d} \tilde{z} \mathrm{~d} s_{h}^{k} \mathrm{~d} s_{e} \\
& \left(\frac{\left(\tilde{p}_{e \tau}+p_{h \tau}^{k}\right) \tilde{z}}{\hbar}-\frac{\left(z s_{h}^{k}+\tilde{z} s_{e}\right)}{\hbar}\right) \\
& \\
& \\
& \\
& \\
& \\
&
\end{aligned}
$$

where the sets $q_{e}, p_{e}\left(q_{h}, p_{h}\right)$ refer to the coordinates and momenta of $M$ electrons ( $M^{\prime}$ holes). Further, a bar (e.g. $\bar{q}$ or $\bar{p}$ ) indicates that the $j$ th electron (or $k$ th hole) is missing in the variable set and $\tilde{p}_{e \tau}$ or $\tilde{p}_{h \tau}$ denote the momentum of the additional $(M+1$ th $)$ electron or $\left(M^{\prime}+1\right.$ th $)$ hole.

\section{Numerical results}

From a mathematical point of view, the $\mathrm{WF} f_{M^{\prime}}^{M}=\tilde{f}^{M, M^{\prime}}$ can be considered as a countervariant tensor of valency two [17]. So the integral equation (23) and its related solution are nothing more than the tensor generalization of the integral equation (9) and the iteration series (11). The possibility to convert a series like equation (11) into a form convenient for probabilistic interpretation allows us to apply MC methods to its evaluation [4-9]. In order to sum up the terms giving the dominant contribution to the WL function in the tensor iteration series, we should generalize the MC approach developed in [4] to sample trajectories in phase space of various 
dimensionalities related to different values of $M$ (number of electrons) and $M^{\prime}$ (number of holes). Formally speaking, $M$ and $M^{\prime}$ independently take values from zero up to infinity, and trajectories are described by the following set of dynamic variables: $M, M^{\prime}$ and the $p q$-trajectory in the phase space of dimensionality $\left(6 M+6 M^{\prime}\right)$. In principle, it is possible to restrict the values of $M$ and $M^{\prime}$ by a certain large number $N_{\max }$. In this case, we should simulate the dynamics of $N_{\max }^{2}$ independent electron-hole systems as following from equation (23) with $\gamma_{\text {rad }}=0$.

To understand the algorithm of sampling trajectories, let us consider the physical meaning of the terms on the rhs of equation (23). The first term, as we mentioned above, describes the classical evolution of the initial quantum WF. As a first test, here we use the approximation which takes into account only the quantum corrections to classical trajectories (position and momentum jumps), which arise from electron-hole recombination, while momentum jumps related to the Heisenberg uncertainty (second term on the rhs of equation $(23)[4,9])$ are neglected. This simplification of the problem allow us to analyse the influence of the terms proportional to $\gamma_{\mathrm{rad}}$. The third term in equation (23) with $f_{M^{\prime}}^{M}$ conserves the numbers $M$ and $M^{\prime}$ and describes the electronhole recombination (photon emission) in one space point and the immediate (photon absorption) creation of an electron-hole pair in another space point. This term describes momentum and space jumps of one electron-hole pair and conserves the virtual momentum of the trajectory and the number of particles in the system. So in our algorithm, it is necessary to break the dynamics of classical virtual trajectories by jumps of this type. As a result of the tensor multiplication in the iteration series, the fourth and fifth terms in equation (23) with $f_{M^{\prime}+1}^{M}$ and $f_{M^{\prime}}^{M+1}$ are responsible for the appearance of the new nonzero tensor elements $f_{M^{\prime}}^{M}$ with smaller values of $M$ and $M^{\prime}$. So these terms describe the evolution of numbers $M$ and $M^{\prime}$, which physically relates to the processes of disappearance of one electron or one hole. To simulate the evolution of the electron-hole systems with $M=M^{\prime}$ giving the main contribution to quantum observables we should introduce two subsequent momentum and space jumps of the classical virtual trajectories resulting in the disappearance of one electron and one hole. So, the WF can be presented in the form of the initial WF contributions of the trajectories with jumps of the above mentioned types. Certainly, to simulate $N_{\max }^{2}$ electronhole systems simultaneously is impossible as it would require excessive computer resources. To overcome this difficulty we use an approximation where instead of the transitions to systems with smaller number of particles $M$ and $M^{\prime}$ and, consequently, smaller density, we increase the volume of the system. Further details of the theory and of our numerical approach will be published elsewhere.

Figure 1 presents first results for the evolution of the electron-hole density due to recombination. The initial WL function $f_{0}$ corresponds to a fully ionized laser-excited electron-hole plasma under conditions of strong Coulomb correlations, $r_{s}=10$. We clearly observe the decay of the carrier number due to recombination confirming the correctness and feasibility of our method.

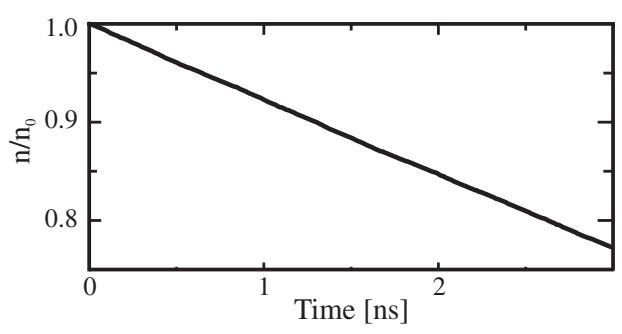

Figure 1. Reduction of the density of laser excited electron hole pairs in a strongly correlated semiconductor with $r_{s}=10$. Density is in units of the initial density $n_{0}$. The excess energy of the initial electron hole plasma is $0.2 \mathrm{Ryd}$

\section{Acknowledgments}

This work was supported by the Deutsche Forschungsgemeinschaft through the Quantum Optics in Semiconductors Research Group, by the Humboldt Foundation and the Max-Planck Society through the Max-Planck Research prize, by the Optodynamics Center of the Philipps-Universität Marburg, and by the Gustav-Hertz prize of the Deutsche Physikalische Gesellschaft. MK acknowledges funding from the Swedish Natural Science Research Council (NFR) and the Göran Gustafssons Stiftelse and CPU time from the Center for Parallel Computers (PDC).

\section{References}

[1] Filinov V S, Bonitz M, Fortov V E and Levashov P R 2001 Phase transition in strongly degenerate hydrogen plasma JETP Lett. 74384

[2] Filinov V S, Bonitz M, Ebeling W and Fortov V E 2001 Thermodynamics of hot dense H-plasmas: path integral Monte Carlo simulations and analytical approximations Plasma. Phys. Control. Fusion $\mathbf{4 3} 743$

[3] Zamalin V M, Norman G E and Filinov V S 1977 The Monte-Carlo Method in Statistical Thermodynamics (Moscow: Nauka)

[4] Filinov V, Medvedev Yu and Kamskyi V 1995 Quantum dynamics and wigner representation of quantum-mechanics J. Mol. Phys. 85711

[5] Filinov V S 1996 Wigner approach to quantum statistical mechanics and quantum generalization molecular dynamics method J. Mol. Phys. 881517

[6] Filinov V S, Lozovik Y E, Filinov A V, Zakharov I E and Oparin A M 1998 Quantum dynamics in canonical and micro-canonical ensembles. Part II. Tunneling in double well potential Phys. Scr. $\mathbf{5 8} 297$

[7] Ciccotti G, Pierleoni C, Capuani F and Filinov V S 1999 Wigner approach to the semiclassical dynamics of a quantum many-body system: the dynamic scattering function of He-4 Comput. Phys. Commun. 121/122 452

[8] Lozovik Yu and Filinov A 1999 Transmission times of wave packets tunneling through barriers Sov. Phys.-JETP $\mathbf{8 8}$ 1026

[9] Filinov V S, Thomas P, Varga I, Meier T, Bonitz M, Fortov V E and Koch S W 2002 Interacting electrons in a one-dimensional random array of scatterers-a quantum dynamics and Monte Carlo study Phys. Rev. B 65 165124

[10] Bonitz M 1998 Quantum Kinetic Theory (Leipzig: Teubner)

[11] Haug H and Koch S W 1994 Quantum Theory of the Optical and Electronic Properties of Semiconductors 3rd edn (Singapore: World Scientific) 
[12] Kira M, Jahnke F, Hoyer W and Koch S W 1999 Quantum theory of spontaneous emission and coherent effects in semiconductor microstructures Prog. Quantum Electron. 23189

[13] Kira M, Hoyer W, Stroucken T and Koch S W 2001 Exciton formation in semiconductors and the influence of a photonic environment Phys. Rev. Lett. 87176401
[14] Tatarskii V 1983 The Wigner representation of quantum mechanics Sov. Phys.-Usp. 26311

[15] Sobol I M and Messer R (Translator) 1975 Monte Carlo Methods (Chicago, IL: University of Chicago Press)

[16] Cohen-Tannoudji C, Dupont-Roc J and Grynberg G 1989 Photons and Atoms 3rd edn (New York: Wiley)

[17] Schouten J A 1951 Tensor Analysis for Physicists (Oxford: Clarendon) 